\title{
Los riesgos y desastres naturales en la cotidianidad de los barrios de lepanto, puntarenas, Costa Rica
}

\author{
Risks and Natural Disasters in the Everyday Life of \\ Lepanto, Puntarenas, Costa Rica
}

Carlos Montero Cascante

Universidad Nacional

OVSICORI

Heredia, Costa Rica

cmontero@una.ac.cr

Susana Espinoza Hernández

Universidad Nacional

OVSICORI

Heredia, Costa Rica

susana12espinoza@gmail.com

Rebeca Lazo Romero

Universidad Nacional

OVSICORI

Heredia, Costa Rica

rebeca.lazo.romero@una.cr

Daniela Campos Durán

Universidad Nacional

OVSICORI

Heredia, Costa Rica

dcd0490@gmail.com

Recibido: 28/02/2018 Aceptado: 20/06/2018

Resumen. El presente artículo nace en el marco del proyecto "Educación para la prevención de riesgos naturales" utilizado como parte del proceso de extensión y docencia de Instituto 
ReVista Universidad en Diálogo • Vol. 8, N. ${ }^{\circ}$ 2, Julio-Diciembre, 2018, pp. 81-98

ISSN 2215-2849 • EISSN: 2215-4752

DOI: http://dx.doi.org/10.15359/udre.8-2.6

de Investigación Observatorio Vulcanológico y Sismológico de Costa Rica OVSICORI-UNA, el cual consiste en el desarrollo de una metodología educativa que busca promover una cultura de prevención de riesgos en el sector educativo formal del país. Mediante el trabajo en conjunto del OVSICORI-UNA con el Programa de Desarrollo Integral de las Comunidades Rurales y Costeras de la Universidad Nacional PDICRC, se aplicó la metodología educativa en prevención de riesgos en las comunidades de Lepanto, Cabo Blanco, Esperanza, Pueblo Nuevo y La Ilusión, pertenecientes al distrito de Lepanto, cantón de Puntarenas con el sector educativo de primaria. Para el desarrollo de este artículo se contó con la información obtenida en los mapas de percepción del riesgo realizados por la población meta, además de ello se llevó a cabo la consulta de la base de datos DesInventar, de la cual se obtuvo información de los eventos naturales que han afectado históricamente al distrito de Lepanto, cuya información abarca el periodo 1988-2015. Aunado a lo anterior, es importante señalar que como parte del proceso se han generado diálogos con las personas que habitan en esta comunidad, quienes destacan las sequías, sismos, deslizamientos, avalanchas e inundaciones, como una problemática de riegos en la zona, debido a estas razones se determina la necesidad de planificar un trabajo conjunto a cerca de la prevención de riesgos y desastres naturales con los niños, niñas y docentes de los Centros Educativos, para que de esta manera la información sea transmitida a la comunidad en general. A través de dicha situación se ha establecido la importancia de fortalecer la participación de los Centros Educativos de Lepanto, como estrategia para guiar a estas personas en el tema, en aras de una mayor sensibilización del contexto real que afrontan, por tanto es importante tomar conciencia de los riesgos que propensos en la zona, así como identificar las causas de los mismos y las formas de mitigarlos o evitarlos, asegurando una mayor integración y seguridad comunitaria, iniciando desde una de la instituciones más importantes: los Centros Educativos.

Palabras claves: prevención, educación, percepción de riesgo, comunidad.

Abstract. This article presents the methodology and the results of the "Education for the prevention of natural risks" project that aims to promote risk prevention in the education sector of Costa Rica. This project is part of the extension and teaching program of the Costa Rican Volcanology and Seismology Observatory of the National University of Costa Rica (OVSICORI - UNA) and is supported by the "Programa de Desarrollo Integral de las Comunidades Rurales y Costeras" (Integral Development Program for Rural and Coastal Communities, PDICRC-UNA) at the National University of Costa Rica. The methodology was implemented in primary schools in the communities of Lepanto, Cabo Blanco, Esperanza, Pueblo Nuevo, and La Ilusión belonging to the district of Lepanto, Puntarenas, Costa 
Rica. The methodology combines a risk perception map prepared by children and with the help of DesInventar database that contains information about the natural events that have affected the district of Lepanto between 1988 and 2015. Another important element of the methodology involves dialogues with the local population about catastrophes such as earthquakes, landslides, avalanches, and floods, which have affected the communities. The data gathered with the methodology point out the benefits of involving children and primary school professors to promote the information about the prevention of natural risks to all the community. This project establishes the importance that the education centers of Lepanto can play to guide communities and to increase their awareness of the natural risks they are facing, as well as to find ways to mitigate them.

Keywords: prevention, education, perception of risk, community.

\section{Introducción}

La comunidad de Lepanto es la cabecera del distrito de Lepanto, el cual corresponde al cuarto distrito del cantón de Puntarenas, que posee una extensión de 420, $46 \mathrm{~km}^{2}$ y limita al norte con el Golfo de Nicoya, al sur y oeste con el cantón de Nandayure y al este con el distrito de Paquera.

El Instituto de Desarrollo Rural (2016) señala que el distrito se caracteriza por ser un territorio rural, cuyas principales actividades económicas están ligadas a la producción agropecuaria, la pesca artesanal y la agricultura. Además, de acuerdo con el censo de población del 2011, el distrito contaba para ese año con un total de 9502 habitantes, de los cuales 4767 eran hombres y 4735 correspondían a mujeres; un 27\% de la población habitaba en área urbana y un $73 \%$ en área rural.

Este territorio además presenta una serie de condiciones hidrográficas y geológicas que generan escenarios de riesgo para las comunidades, tal como lo son las recurrentes inundaciones, las cuales se deben a la ocupación de las planicies de inundación y al desarrollo urbano en forma desordenada y sin ninguna planificación, es decir, al margen de las leyes que regulan el desarrollo urbano y ambiental (INDER, 2015).

Asimismo, la cercanía a la zona de subducción de las placas Coco y Caribe en el sector de Nicoya lo vuelve un territorio vulnerable a eventos sísmicos, tal como los ocurridos en el año 1992 o el terremoto de Nicoya en el año 2012. 
ReVista Universidad en Diálogo • Vol. 8, N. ${ }^{\circ}$ 2, Julio-Diciembre, 2018, pp. 81-98

ISSN 2215-2849 • EISSN: 2215-4752

DOI: http://dx.doi.org/10.15359/udre.8-2.6

Lepanto presenta un escenario de vulnerabilidades, pero además cuenta con fortalezas y oportunidades que requieren ser más desarrolladas, un ejemplo de esto es el río Lepanto, que determina ser un beneficio para la comunidad, ya que gracias a este elemento es posible que la zona cuente con agua potable mediante el sistema de bombeo; el manglar y las diferentes quebradas cerca de las viviendas representan para la zona una debilidad, así como el hecho de no contar con las medidas necesarias para regular una situación de emergencia.

A pesar de que la comunidad cuenta con un Comité Local y Municipal de Emergencias, no posee un plan concreto de preparativos y respuesta ante una emergencia; sin embargo, en el documento del Plan Estratégico del Consejo Municipal se menciona dentro de la normativa ambiental la necesidad de exigir el cumplimiento de las leyes ambientales (entre estas la creación de un plan de emergencia). Es importante señalar que ese documento no es creado solo para la comunidad de Lepanto, es el Plan Estratégico de todo el distrito, por este motivo es necesario identificar los riesgos a los que está expuesta a vivir esta zona rural costera e incentivar desde el sistema educativo el desarrollo de planes familiares de prevención.

Existen dos tipos de modelos relacionados con los desastres, uno de ellos es el modelo de gestión de riesgos, que consiste en aquellos desastres que se pueden prevenir, pero no evitarse. Otro modelo es el que atiende el desastre, es el más tradicional, ya que reacciona post desastre, cuando la comunidad es atendida por las instituciones locales y nacionales, este último modelo es el que actualmente se distingue en la comunidad lepanteña.

Condiciones como las anteriores generan situaciones de riesgo para las comunidades, donde estas mismas, debido a sus prácticas culturales de ocupación del territorio, propician la ocurrencia de eventos que resultan perjudiciales para la población. Tal como señalan Aguilar y Brenes (2008), la comunidad como actor social juega un papel en tres direcciones, por un lado como generadora de riesgo, por otro como receptora de consecuencias y además como agente de cambio, capaz de realizar acciones para abordar su situación de riesgo.

Sin embargo, la percepción de riesgos juega un papel fundamental en la construcción individual y comunal del espacio, ya que esto puede determinar posibles soluciones para accionar colectivamente ante las distintas dinámicas presentes en las comunidades. 


\section{Metodología}

Para llevar a cabo la presente investigación se realizó una caracterización histórica de eventos naturales que han afectado a la población del distrito de Lepanto, para ello se consultó a la comunidad y a la base de datos de DesInventar, la cual se caracteriza por registrar pérdidas y daños de diversas magnitudes originados por la ocurrencia de eventos naturales o antrópicos.

La información consultada en la base de datos DesInventar correspondió al periodo comprendido entre los años 1988 y 2015, esta además es de carácter oficial, ya que fue suministrada por la Comisión Nacional de Prevención de Riesgo y Atención de Emergencias (CNE), así como informes periodísticos del periódico La Nación.

Asimismo, los eventos naturales que se consideraron fueron: actividad volcánica, alud, aluvión, avenida torrencial, deslizamiento, huracán, inundación, lluvias, marejadas, sequía, sismo, tormenta eléctrica, tornado y tsunamis.

Con la información obtenida en la base de datos DesInventar se logró determinar para el periodo 1988-2015 cuáles fueron los tipos de eventos registrados, su ubicación, así como los años y meses de mayor afectación.

Además, se llevaron a cabo talleres de capacitación en el tema educativo en prevención de riesgos naturales con el Comité Local de Emergencias de Lepanto y los centros educativos de primaria presentes en el área de estudio. Donde se realizaron mapas de percepción de riesgos. Dichos mapas de percepción de riesgo se llevaron a cabo aplicando la guía didáctica para docentes y personas facilitadoras "Introducción a la prevención de riesgos naturales", herramienta educativa que permite obtener información espacial sobre la percepción de los riesgos naturales a los que las personas se sienten expuestas en sus viviendas y comunidades.

En esta metodología educativa los riesgos que se contemplan son: terremotos, erupciones volcánicas, inundaciones, huracanes, deslizamientos, tornados, tsunamis y otros riesgos.

Finalmente se realizó un análisis comparativo entre la información histórica de eventos y la percepción de la población, con el fin de determinar la relación existente entre los diferentes eventos que han impactado a las comunidades y la percepción de la población en cuanto a los riesgos. 
ReVista Universidad en Diálogo • Vol. 8, N. ${ }^{\circ}$ 2, Julio-Diciembre, 2018, pp. 81-98

ISSN 2215-2849 • EISSN: 2215-4752

DOI: http://dx.doi.org/10.15359/udre.8-2.6

\section{Resultados}

Es importante reflexionar sobre la prevención de riesgos y desastres a partir de los riesgos a los que se encuentra expuesta la comunidad, así como su causa y efecto, por lo tanto, en la consulta realizada a los diferentes barrios de la comunidad se destacan las siguientes causas:

- La falta de integración y de organización por parte de la comunidad en torno al tema de riesgos ambientales

- Amenazas provocadas por el ser humano, como por ejemplo la tardanza en la construcción de puentes y su mala planificación, cuando son tan importantes dentro de la zona. Un alto grado de vulnerabilidad social expresado en el deterioro de infraestructura comunal, importantes vías de comunicación (puentes)

- Falta de apoyo institucional en la promoción de la gestión de riesgo, específicamente en la atención de emergencias

- Desconocimiento de planes para la reducción de riesgos

- Escasa intervención en el tema de riesgos por parte de la población juvenil, con excepción de los y las jóvenes del grupo "4S", del barrio Rosa Claus de Lepanto, quienes han mostrado mayor participación en el tema durante los años 2012-2013. Actualmente, el grupo no se encuentra consolidado.

Los problemas centrales de estas causas se determinan a través de dos factores relevantes y con la necesidad de tratarlos de manera colectiva, para este caso se habla desde una visión dialéctica de las inundaciones que se dan por el tipo de zona y las vulnerabilidades que se presentan, para lo cual el impacto puede llegar a ser bastante fuerte; en algunas temporadas surge una mayor afectación que en otras.

La comunidad compara la gravedad de las inundaciones del 2010, 2011 y 2012, años en los que la cantidad de lluvia ocasionó desastres, mientras que en la actualidad se presenta principalmente el fenómeno de la sequía, no obstante, en temporada de invierno algunos barrios como Valle Fresco, Pueblo Nuevo y Rosa Claus suelen ser los más afectados. 
Abeldaño (2015) enfatiza en la importancia de

Indagar en las respuestas emocionales frente a un desastre, como un elemento necesario para el abordaje integral del estrés psicoemocional. También se considera importante la evaluación de la severidad del impacto y el análisis de las capacidades humanas y de los recursos de gestión local y en lo concerniente a la percepción de un desastre, es necesario indagar entre los factores relacionados con la edad, género, salario, tipo de residencia, empleo, educación, entre otros.

Debido a las causas mencionadas, se generan efectos tales como las preocupaciones e impactos psicosociales que enfrenta la población día tras día con respecto a esta situación, por ejemplo: las enfermedades en épocas de invierno, la baja calidad de servicios básicos, las instituciones que se apegan a la Ley Marítimo Terrestre y despojan a la comunidad de sus viviendas sin brindarles solución, el mal diseño de puentes que son fuente de comunicación con otros pueblos, la inadecuada planificación e inversión de diques que no funcionan, malos olores y enfermedades por la contaminación y desaprovechamiento de residuos.

Es por esto que resulta fundamental destacar la generación de redes comunitarias a partir de las respuestas psicosociales que, a pesar de tener las características incipientes por la naturaleza del evento, tienen la potencialidad de facilitar el trabajo multisectorial para la intervención en la emergencia.

Los puntos señalados anteriormente describen una comunidad con importantes necesidades, y se hace hincapié en que para manejar dicho escenario esta población requiere seguir incentivando la integralidad y la participación ciudadana en temas relacionados con la prevención y los planes de emergencia, permitiendo el fortalecimiento del Comité Comunal de Emergencias, así como los demás comités que conforman la comunidad.

Además, es importante que los centros educativos capacitados en educación para la prevención de riesgos naturales propongan un seguimiento de trabajo, ya que cada año ingresan nuevos y nuevas estudiantes y se busca que la persona participante, más que ser una receptora de información, sea partícipe activa del proceso, en donde cada docente interprete la metodología de trabajo como una forma más factible para transmitir a sus 
ReVista Universidad en Diálogo • Vol. 8, N. ${ }^{\circ}$ 2, Julio-Diciembre, 2018, pp. 81-98

ISSN 2215-2849 • EISSN: 2215-4752

DOI: http://dx.doi.org/10.15359/udre.8-2.6

respectivos estudiantes la importancia del tema y que posteriormente sean intermediarios/as de información, transfieran el aprendizaje a sus propias familias y estas a las demás personas de la comunidad.

\section{Registro histórico de eventos en el distrito de Lepanto, periodo 1988-2015}

De acuerdo con la base de datos DesInventar, se determinó que entre los años 1988 y 2015 se registraron un total de 57 eventos, de los cuales 50 correspondieron a inundación, cuatro a deslizamiento, un incidente por lluvias y otro por sismo. Los años que más eventos presentaron fueron el 2011, 2008 y 2007, tal como se muestra a continuación.

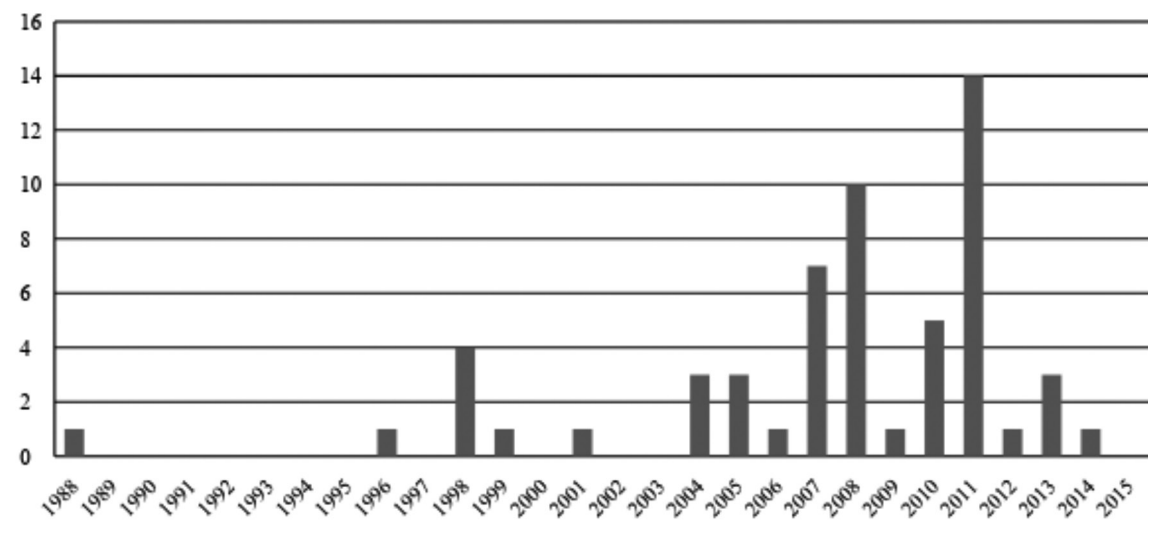

Figura 1. Cantidad de eventos por año, periodo 1988-2015.

Fuente: elaboración propia, según los datos reportados por DesInventar, 2016.

De acuerdo con el gráfico anterior, se puede apreciar que el año 2011 es el que históricamente ha presentado mayor incidencia de eventos, con un total de catorce, todos correspondieron a inundación y estuvieron asociados a sistemas de baja presión y a la depresión tropical № 12 en el mes de octubre, lo cual provocó cuatro personas damnificadas y dos viviendas dañadas, donde las principales comunidades afectadas fueron Cabo Blanco, barrio El Jardín, Cedros, El Coto, Lepanto, San Pedro y San Blas (DesInventar, 2016). 
Por su parte en el año 2008 se presentaron diez eventos, de los cuales ocho fueron inundación y dos deslizamientos, asociados a fuertes lluvias principalmente en los meses de mayo y octubre, y que afectaron a las comunidades de Jicaral, La Esperanza, Cabo Blanco y Lepanto.

En el año 2007 se dieron siete eventos donde cinco de ellos correspondieron a inundación, los cuales afectaron a las comunidades de Cabo Blanco, Jicaral y Lepanto.

\section{Datos de la entrevista para el rescate de la memoria histórica}

En tres de las cinco escuelas, el personal docente realizó el proceso completo de la metodología con sus estudiantes, así como los informes. En el caso de Cabo Blanco y Lepanto centro se ejecutó la mitad de la misma con registro de informes.

A continuación, se presenta una tabla en la cual se desglosa la información más relevante recopilada de la entrevista para el rescate de la memoria histórica, la cual corresponde a una guía didáctica para docentes y es un medio para compartir experiencias, información y conocimiento en materia de prevención de riesgos, favoreciendo el diálogo intergeneracional y de saberes.

Esta entrevista busca el diálogo intergeneracional entre las personas más jóvenes y las personas adultas mayores, para que a través de su experiencia puedan difundir su conocimiento, con el fin de generar una memoria histórica y que la población infantil identifique las dinámicas propias de su comunidad hace cincuenta años atrás, lo que significa en el presente y lo que podría llegar a ser generando una visión futura, según la percepción del estudiantado. Dentro del Marco de Sendai para la Reducción del Riesgo de Desastres 2015-2030 se menciona como un principio para la prevención de riesgos:

Si bien los factores que pueden aumentar el riesgo de desastres pueden ser de alcance local, nacional, regional o mundial, los riesgos de desastres tienen características locales y específicas que deben comprenderse para determinar las medidas de reducción del riesgo de desastres (p. 13).

Es por lo anterior que la actividad requiere ser compartida y evaluada en una sesión plenaria con el estudiantado y que se pueda, de ser posible, invitar a alguna persona de la comunidad para que intercambie sus experiencias relacionadas con un desastre vivido en la comunidad o en otros sitios a lo largo de su vida. 
ReVista Universidad en Diálogo • Vol. 8, N. ${ }^{\circ}$ 2, Julio-Diciembre, 2018, pp. 81-98

ISSN 2215-2849 • EISSN: 2215-4752

DOI: http://dx.doi.org/10.15359/udre.8-2.6

A continuación en la Tabla 1, se muestra el centro educativo participante de la capacitación, que además ha llevado a cabo todos los procesos de la metodología; también se detalla el número de estudiantes participantes, el rango de edad de las personas que fueron entrevistadas por el estudiantado, el tipo y lugar del evento que han vivido estas personas, los principales daños presentados una vez ocurrido el fenómeno y finalmente cuál fue la atención que recibieron. Estas son algunas interrogantes importantes que se presentan en la entrevista o guía 1 .

Con respecto a la tabla 1 , se señala que cuando han ocurrido eventos masivos en la comunidad se realizan algunos cambios en la cotidianidad de la población, se tardan días sin poder trabajar, no se puede asistir a los centros de educación por falta de paso, surgen enfermedades, se trabaja en la restauración del paso en la calle, trabajos en las casas y se trabaja en el desastre de las fincas que algunos eventos han ocasionado.

Según el Marco Sendai (2015-2030),

La reducción del riesgo de desastres requiere la implicación y colaboración de toda la sociedad. Requiere también empoderamiento y una participación inclusiva, accesible y no discriminatoria, prestando especial atención a las personas afectadas desproporcionadamente por los desastres, en particular las más pobres. Deberían integrarse perspectivas de género, edad, discapacidad y cultura en todas las políticas y prácticas, y debería promoverse el liderazgo de las mujeres y los jóvenes. En este contexto, debería prestarse especial atención a la mejora del trabajo voluntario organizado de los ciudadanos (p. 14).

De acuerdo con esto, se resumen las recomendaciones generadas por parte del estudiantado, reflejando la importancia de mantener la precaución precisa al construir las viviendas o edificaciones, considerando las medidas de prevención necesarias de acuerdo con las dinámicas y condiciones propias de la comunidad, ya que la finalidad está orientada hacia la búsqueda de soluciones para evitar que año tras año se repita la misma situación, y de este modo disminuir la vulnerabilidad de la población. Además, se rescata que estos riesgos representan el inadecuado uso que le da el ser humano a este tipo de medidas. 
Revista Universidad en DiÁlogo • Vol. 8, N. ํㄹ 2, Julio-Diciembre, 2018, pp. 81-98

ISSN 2215-2849 • EISSN: 2215-4752

DOI: http://dx.doi.org/10.15359/udre.8-2.6

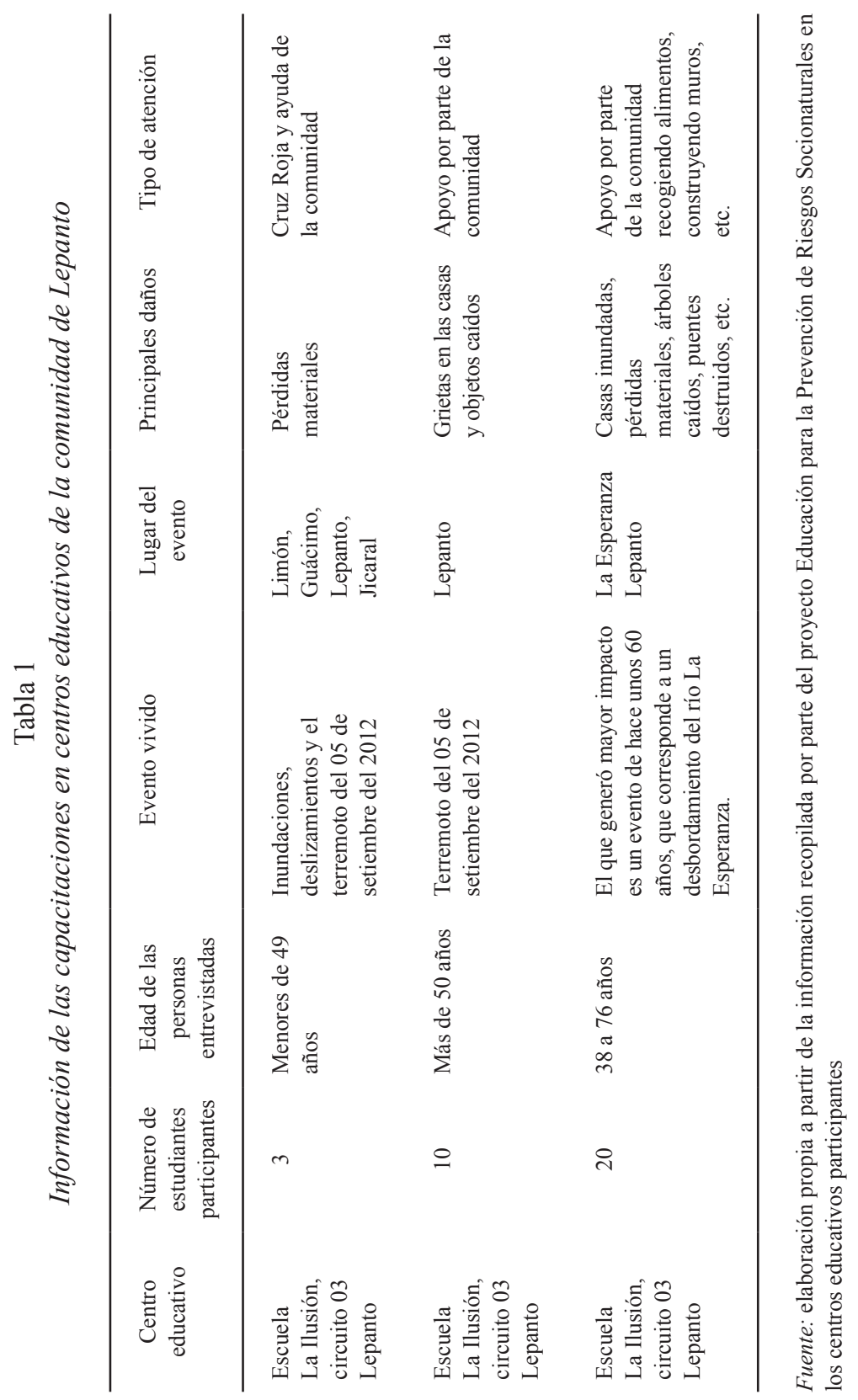


Revista Universidad en Diálogo • Vol. 8, N. ํㄹ Julio-Diciembre, 2018, pp. 81-98

ISSN 2215-2849 • EISSN: 2215-4752

DOI: http://dx.doi.org/10.15359/udre.8-2.6

Tal como lo plantea De Romilly (1998), citado por Molano (2007): "Nadie puede vivir sin recordar y nadie puede vivir tampoco sin los recuerdos de la historia (...) la historia está allí orientando nuestros juicios a cada instante, formando nuestra identidad, determinando la fuente y toma de conciencia de nuestros valores" (p. 84).

De manera tal que darle un lugar a la memoria histórica comunitaria facilita el desarrollo de la identidad y la imagen comunitaria, que son aspectos sutiles que nutren su accionar, favoreciendo la comprensión de sus propios procesos organizativos y de gestión local para el logro de las metas colectivas en su territorio, tomando en cuenta sus recursos y sus riesgos.

Salas (2007) deduce lo siguiente,

De los quince países del mundo con mayores porcentajes de superficie de sus territorios expuestos a tres o más tipos de riesgos, cinco de los países "un tercio del total mundial" se encuentran en el 'Área de Estudio': Costa Rica, Guatemala, El Salvador, Panamá y Nicaragua. Todos, con tres tipos de riesgos de fenómenos naturales aunque con variaciones notables entre sí, tanto en porcentaje de área como de población expuesta (p. 6).

Dando énfasis a la cita anterior, se menciona que Costa Rica se encuentra en la posición número siete de países de Centroamérica y el Caribe con mayores porcentajes de territorio expuesto a dos o más riesgos (seleccionados entre los sesenta países del mundo con mayores riesgos) y de los quince países del mundo con mayores áreas porcentuales de sus territorios expuestos a tres o más tipos de riesgos, es decir, es un país expuesto a diferentes riesgos. Por lo que es necesario fomentar en estos procesos la participación del mayor porcentaje de población para que se logre una comunicación asertiva y la sustentabilidad de los planes locales y familiares enfocados en la prevención de riesgos y desastres, tomando en cuenta tanto a la población infantil y joven como a la adulta y adulta mayor, sin ningún tipo de discriminación, ya que la reducción del riesgo requiere la preparación y cooperación de toda la sociedad, especialmente si se trata de zonas con altos indicadores de pobreza en forma de asentamientos humanos precarios, de soluciones habitacionales en terrenos vulnerables, viviendas estructuralmente deficientes, falta de participación activa de la comunidad y escaso apoyo por parte de las instituciones del Estado. 


\section{Datos del Plan Familiar de Prevención}

Para conocer más detalladamente el contenido de esta guía es importante mencionar algunas recomendaciones e interrogantes que se analizan en conjunto con la familia:

Tabla 2

\section{Plan Familiar de Prevención}

\begin{tabular}{|l|}
\hline Número de personas que viven en la casa. \\
\hline Tipo de riesgos a los que la familia considera estar expuesta (se agregan ejemplos). \\
\hline Observar e identificar zonas de alta pendiente o cercanas a ríos, entre otras \\
\hline Tipos de materiales con que está construida la casa. \\
\hline Disponibilidad de planos y revisar si concuerdan con la construcción final. \\
\hline Si no existen planos, identifique las debilidades de la construcción. \\
\hline Identificar muebles u objetos colgantes \\
\hline Revisar que los pasillos se encuentren despejados. \\
\hline Comentar sobre la importancia de tener reserva de artículos útiles \\
\hline ¿Cómo localizar en caso de una emergencia a las personas integrantes de la familia? \\
\hline Identificar zonas de evacuación en caso de una emergencia \\
\hline Señalar a las personas responsables de cada actividad por resolver \\
\hline ¿Participaron todas las personas del grupo familiar en completar el plan? \\
\hline ¿Acordaron plazos para revisar el plan familiar de prevención? \\
\hline
\end{tabular}

Fuente: Guía \#2, Plan Familiar de Prevención.

Para el caso de las familias de los centros educativos trabajados, se han destacado las siguientes respuestas ante las variables mencionadas anteriormente: 
- La mayoría de las familias (de cinco centros educativos) de la zona mencionan que sus viviendas no poseen planos y que consideran que no están construidas adecuadamente, ya que en época de fuertes lluvias se introduce el agua a sus hogares debido al desnivel de la vivienda con respecto a la calle. En eventos tales como temblores se tienden a hacer grietas en las paredes.

- También señalan que no cuentan con botiquines de emergencia, sin embargo, comúnmente poseen linternas.

- En la Escuela de La Esperanza se trabajó en simulacros en caso de emergencia y la maestra gestionó la compra de carteles para la señalización para orientar a la población infantil sobre cuáles son los sitios de evacuación hacia las zonas de seguridad.

- La mayoría de las familias consideran estar expuestas a inundaciones, por el desbordamiento de quebradas, algunas a deslizamientos, a terremotos y a incendios forestales.

- En la mayoría de los planes hubo participación de toda la familia y según la evaluación se encuentran en estado intermedio, donde se debe revisar nuevamente el plan y corregir los aspectos que sean convenientes, según lo sugerido en la autoevaluación de este instrumento.

- En las distintas escuelas al concluir el proceso se realizó una reunión con padres y madres de familia, con la finalidad de darles a conocer las temáticas y procedimientos desarrollados con sus hijos e hijas, también se trató de propiciar un espacio de intercambio de conocimientos, sensibilización y reflexión acerca de la importancia de la prevención de riesgos en el diario vivir y la forma en la cual esto podría apoyar en el desarrollo de las comunidades fomentando la integración de temas y acciones.

- Además, al momento de realizar la intervención comunitaria es necesario tomar en cuenta que el fomento de la integración de las personas participantes en el desarrollo, ejecución y evaluación del proceso es indispensable para estimular y fortalecer el sentido de comunidad, identidad y pertenencia a su territorio. De esta manera se sentirán capaces de buscar soluciones a las problemáticas que se les presentan, evitando caer en la dependencia a los y las profesio- 
nales que facilitan los procesos comunitarios, tal como lo plantean Hombrados y Gómez (2001), de manera tal que se les faculta para que se sientan competentes para que hagan sus propias gestiones.

\section{Percepción de riesgos naturales en el distrito de Lepanto}

Para la realización de los mapas de percepción del riesgo en el distrito de Lepanto se trabajó con el personal docente y el estudiantado de las escuelas $\mathrm{La}$ Esperanza, La Ilusión, Lepanto centro, Pueblo Nuevo y Cabo Blanco. En estas escuelas se trabajó con un total de 35 niños y niñas entre los seis y doce años.

Los riesgos naturales que identificó el Comité Local de Emergencias de Lepanto y la población estudiantil de primaria en sus mapas de percepción fueron los siguientes.

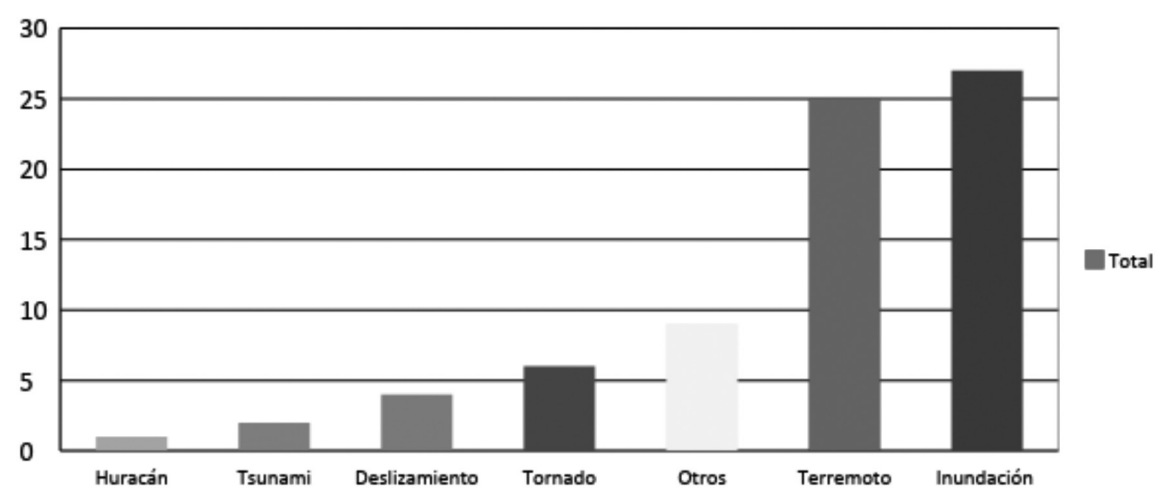

Figura 2. Percepción de riesgos en las comunidades de La Esperanza, La Ilusión, Cabo Blanco y Lepanto

Fuente: elaboración propia

\section{Cogestión del Comité Comunal de Emergencias}

A partir del proceso de acompañamiento y capacitaciones impartidas para el Comité Comunal de Emergencias de Lepanto, se ha logrado la nueva conformación y juramentación del Comité Comunal de Emergencias de Lepanto. Ha alcanzado una comunicación activa para generar alianzas con organizaciones relacionadas al tema de emergencias, y para esto el grupo se reúne mensualmente. 
ReVista Universidad en Diálogo • Vol. 8, N. ${ }^{\circ}$ 2, Julio-Diciembre, 2018, pp. 81-98

ISSN 2215-2849 • EISSN: 2215-4752

DOI: http://dx.doi.org/10.15359/udre.8-2.6

El Comité ha recibido materiales que fueron solicitados a la Cruz Roja Americana, tales como: botas, linternas, megáfonos y palas; actualmente cuentan con un proyecto de Sistema de Alerta Temprana en Montaña Grande, Lepanto y Valle Fresco.

Como lo plantea el Programa Medio Ambiente, Gestión del Riesgo y Emergencias (MAGRE)-Caritas Chile, "un horizonte deseado es lograr cambios sustentables en las condiciones de vida de las comunidades, logrando así que tanto éstas como las minorías activas sean autónomas, activas y empoderadas, aumentando su capacidad de resiliencia" (p. 28), permitiendo el fortalecimiento de las capacidades de acción de las comunidades, lo que implica el conocimiento, la organización y la puesta en práctica, debido a que son las comunidades las que conviven con los riesgos y las emergencias.

Dentro del marco de la organización, el Comité se ha encargado de buscar alternativas para participar en foros, también ha colocado señalizaciones en la comunidad, ha creado un Plan Comunitario de Emergencias y cuenta con rutas de evacuación que ha logrado mediante la búsqueda de apoyo de otras instituciones con el propósito de generar redes y alianzas estratégicas. Para esto se ha diseñado un mapa de evacuación en caso de una inundación o maremoto, con el fin de colocarlo en espacios claves de la comunidad y se han establecido recomendaciones relacionadas con las responsabilidades comunitarias en caso de una emergencia para compartir la información con las personas de la localidad.

Los pasos que el grupo debe realizar en caso de una emergencia son realizar una inspección en bicicleta, posterior al reporte de un desastre, y paralelamente reportar la información al 911.

En términos generales, el grupo ha logrado una mayor cogestión comunitaria en temas de prevención de emergencias de acuerdo con las capacitaciones brindadas y el seguimiento del trabajo participativo que se ha venido desarrollando desde el 2014. La frase que utilizan como grupo es: "No hay mayor placer que servir", y vinculado a esta frase mencionan la importancia de realizar rendiciones de cuentas a la comunidad, para cortar toda brecha de comunicación entre la población y el Comité, para esto además visitan cada vivienda para dar a conocer los derechos y deberes que posee la localidad en la prevención de riesgos y desastres. 


\section{Conclusiones}

El trabajo se ha enfocado en plantear capacitaciones para fortalecer el conocimiento del tema en educación hacia la prevención de riesgos y desastres naturales en los centros educativos, considerando el sentido de pertenencia que posee la población analizada hacia el lugar en el que habita.

Sin embargo, es importante rescatar que el enfoque que han generado estas visitas ha sido crear una sensibilidad en el tema de riesgos socionaturales y desastres, donde se incentiva la prevención y la atención ante los mismos, dirigido hacia la reflexión en familia, por ejemplo: a quién buscar en caso de una emergencia, si la vivienda se encuentra en buen estado, qué tipo de afectaciones ha vivido la comunidad o cuáles podría enfrentar, cuáles espacios corresponden a peligros, o bien cuáles son las rutas de evacuación, entre otras interrogantes.

Esto es parte de lo que se ha trabajado con los centros educativos y con la comunidad, más aun tomando en cuenta que es una zona con altos riesgos que la población misma ha detectado y que posee una serie de factores que podrían convertirse en riesgos de gran magnitud si no son debidamente controlados.

Desde una visión dialéctica del riesgo, la situación social de pobreza y el uso del suelo se constituyen como amenazas y potenciadores de vulnerabilidades, esto debido a que dentro de las estrategias de sobrevivencia se encuentra la construcción de viviendas y centros educativos cerca de ríos, manglares, quebradas y deslizamientos y cerca de plantaciones de meloneras que afectan la salud de los niños y las niñas (caso de la Escuela Cabo Blanco), entre otros.

Este tipo de metodologías son amoldadas según el tipo de población con el que se desea trabajar, por lo tanto es importante implementar su uso en otros grupos organizados de la comunidad, como por ejemplo comités cantonales o distritales, para que el tema de prevención sea tratado con toda la población, que niños y niñas, jóvenes y personas adultas puedan utilizar el mismo proceso y no requieran una institución para abordarlo, sino que se transmita la información mediante su conocimiento adquirido en esta metodología, con un sentido de cogestión comunitaria y educativa. 
ReVista Universidad en Diálogo • Vol. 8, N. ${ }^{\circ}$ 2, Julio-Diciembre, 2018, pp. 81-98

ISSN 2215-2849 • EISSN: 2215-4752

DOI: http://dx.doi.org/10.15359/udre.8-2.6

\section{Referencias}

Abeldaño, R. (2015). En R. Abeldaño, M. Lucchese y A. Fernández (coord.), Percepción del desastre y respuestas psicosociales en la comunidad de Tartagal (Argentina): aproximaciones cualitativas a partir del alud de 2009. Escuela de Salud Pública. Facultad de Ciencias Médicas, Universidad Nacional de Córdoba, Argentina.

Aguilar, M. y Brenes, G. (2008). La percepción de riesgo como herramienta para la gestión del riesgo. Aportes para la cogestión comunitaria. Caso de comunidad de Sixaola, Limón, Costa Rica.

DesInventar (2016). Sistema de inventario de efectos de desastres. Recuperado de http://www.desinventar.org/es/database

Hombrados, M. y Gómez, L. (2001). Potenciación en la intervención comunitaria. Intervención Psicosocial, 10(1), 55-69. Recuperado de http://www.copmadrid.org/webcopm/publicaciones/social/68007.pdf

Instituto de Desarrollo Rural (2016). Política de Estado para el Desarrollo Rural Territorial Costarricense (PEDRT) 2015-2030. Recuperado de http://www.inder.go.cr/acerca_del_inder/politicas_publicas/documentos/ PEDRT-2015-2030-Resumen.pdf

Molano, O. (mayo, 2007). Identidad cultural un concepto que evoluciona. Revista Opera, (7), 69-84. Recuperado de http://revistas.uexternado. edu.co/index.php/opera/article/view/1187.

Paz, L., Pérez, T. y Briones, S. (2015). Prácticas de gestión local del riesgo de desastre: hacia la co-construcción de comunidades resilientes. Programa Medio Ambiente, Gestión del Riesgo y Emergencias (MAGRE), Caritas Chile, Conferencia Episcopal de Chile.

Salas, S. (2007). Vulnerabilidad, pobreza y desastres 'socionaturales' en Centroamérica y el Caribe. Instituto de Ciencias de la Construcción Eduardo Torroja (CSIC).

United Nations (2015). Marco de Sendai para la Reducción del Riesgo de Desastres 2015-2030. 